\title{
Ein Fall von «Sterbefasten» wirft Fragen auf
}

\section{Jan Gärtnera , Luzius Müller}

a PD Dr. med., leitender Arzt des Palliativzentrums Hildegard, Facharzt Anästhesie, Intensivmedizin, interdisziplinärer Schwerpunkt Palliative Care, spezielle Schmerztherapie; ${ }^{b}$ Pfr. Dr. theol., Koordinator der Ethikkommission am Bethesda, Dozent für Medizinethik an der Universität Basel und am Bildungszentrum Gesundheit

\begin{abstract}
Der Freiwillige Verzicht auf Nahrung und Flüssigkeit (FVNF; auch «Sterbefasten») wurde in der jüngeren Vergangenheit in diversen Publikationen wiederholt als «milde» Form der Lebensbeendigung beschrieben. Der dargestellte Fall zeigt, dass der FVNF aber auch mit komplexen klinischen, juristischen, versorgungstechnischen und ethischen Herausforderungen verbunden sein kann.
\end{abstract}

\section{Fallbeschreibung}

Mit der Bitte um Übernahme eines betagten, gebrechlichen Patienten trat ein externes Spital an ein Palliativzentrum heran. Der Patient und sein einziger Angehöriger (Neffe) wünschten diese Übernahme dringend, da der Patient einen ausgeprägten Todeswunsch habe. Der Patient wollte bei einem FVNF palliativ optimal begleitet werden. Im zuweisenden Spital war von den mitbehandelnden Fachkollegen der Alterspsychiatrie

Tabelle 1: Freiwilliger Verzicht auf Nahrung und Flüssigkeit (FVNF):

praxisrelevante Aspekte.

\section{FVNF stellt eine Form des Suizids dar.}

FVNF kann mit erheblichen Belastungen der betroffenen Patienten einhergehen, entsprechende Aufklärung der Patienten und Vorausplanung sind unumgänglich.

FVNF kann insbesondere bei Patienten ohne fortgeschrittene und zum Tode führende Erkrankungen mit komplexen Herausforderungen für die betreuenden medizinischen und pflegerischen Fachpersonen einhergehen.

Hunger und Durst unter FVNF sind keine Indikationen zur Sedation.

Lindernde Massnahmen in der Begleitung des FVNF stellen keine Form der Suizidbeihilfe dar, dennoch werden sie von betreuenden Fachpersonen teilweise als solche interpretiert, was zu einer Belastungssituation (moral distress) führen kann.

- Das "Anorexie-Kachexie»-Syndrom im Rahmen fortgeschrittener zum Tode führe der Erkrankungen ist keine Form des FVNF, sondern Teil des normalen Krankheitsverlaufes.

- Die Begleitung des FVNF an sich ist aus Sicht der Autoren keine Indikation für die spezialisierte Palliative Care, jedoch kann aufgrund entstehender Symptome und Probleme bei einigen Patienten eine Mitbehandlung notwendig werden.

- Der alleinige Verzicht auf Nahrung ist in der Regel mit einem langsameren und weniger komplikationsträchtigen Verlauf verbunden als der gleichzeitige Verzicht auf Flüssigkeit und Nahrung.

Der praktische Umgang mit Fragen des FVNF erfordert die Erarbeitung institutioneller Rahmenbedingungen ( Standards») in den Spitälern und Pflegeeinrichtungen.

Der Umgang mit Fragen des FVNF erfordert in jedem Einzelfall eine sorgsame und kollegiale Teamkultur im Umgang mit den unterschiedlichen ethischen Schwerpunktsetzungen der einzelnen Fachpersonen. kein Hinweis für das Vorliegen einer Depression oder anderen Psychopathologie als Ursache des Todeswunsches festgestellt worden.

Da (i) der Wunsch nach Sterbefasten kein Zuweisungsgrund für eine spezialisierte, stationäre Palliative Care darstellt, (ii) keine weiteren Symptome (z.B. Schmerzen, Luftnot usw.) oder psychosozialen Belastungen vorlagen und (iii) der Patient nicht unter einer fortgeschrittenen und zum Tode führenden Erkrankung litt, wurde die Anfrage vom Palliativzentrum abgelehnt. Nach wiederholten Interventionen des zuweisenden Spitals, des Patienten und seines Angehörigen wurde der Übernahme des Patienten jedoch nach längerer Diskussion unter folgender Überlegung zugestimmt: Als Hauptindikation für den Eintritt wurde der ausgeprägte Sterbewunsch deklariert. Abgeklärt werden sollte eine bisher unerkannte körperliche, psychologische, soziale oder spirituelle Belastung des Patienten als Ursache für den Todeswunsch, um diese dann gegebenenfalls behandeln zu können. Im Rahmen des stationären Aufenthaltes erfolgte eine ausführliche und wiederholte multiprofessionelle und interdisziplinäre Exploration des Patienten. Wie im vorbehandelnden Spital konnten keine Hinweise für das Vorliegen einer psychiatrischen Erkrankung ausgemacht werden. Der Patient berichtete eindrucksvoll aus seiner Biographie und vom Wunsch nach Lebensbeendigung. Er sei in verschiedenen Heimen aufgewachsen, wo er sich meist sehr allein und immer wieder «abgeschoben» vorkam. Dieses "Abgeschobenwerden" habe sich wie ein roter Faden durch sein Leben gezogen. Seit einigen Jahren pflegte er eine Beziehung zu einer Frau, die im fernen Ausland domiziliert ist. Diese Beziehung habe seinem 
Leben Sinn gegeben. Aufgrund seiner zunehmenden Gebrechlichkeit habe er diese Beziehung nun nicht mehr in befriedigender Weise fortführen können. So sei ihm der Lebenssinn abhandengekommen.

Der Patient begann im Palliativzentrum mit dem FVNF. Dieser wurde, wie in solchen Fällen üblich, vom betreuenden Team weder unterstützt noch moralisch kritisiert, sondern dem Selbstbestimmungsrecht des Patienten gemäss respektiert.

Der Patient beklagte unter Tränen, dass er selbst in seiner letzten Lebensphase nicht an einem Ort bleiben dürfe, wo er sich "daheim» fühle.

Da aus Sicht des behandelnden Zentrums die Begleitung des FVNF an sich keine Indikation für die spezialisierte, stationäre Palliative Care darstellte, wurde mit der Planung des Übertritts in eine Pflegeeinrichtung begonnen. Der Patient fühlte sich im Palliativzentrum jedoch ausgesprochen wohl und nach eigenem Bekunden "erstmals angenommen", so dass er die bevorstehende Verlegung als ein erneutes "Abgeschobenwerden» empfand. Er beklagte unter Tränen, dass er selbst in seiner letzten Lebensphase nicht an einem Ort bleiben dürfe, wo er sich "daheim» fühle. Nach wiederholten Gesprächen mit dem Patienten und im multiprofessionellen Team wurde daher schliesslich von einer Verlegung des Patienten abgesehen.

Es folgten Phasen, in denen der Patient (möglicherweise als Folge der zunehmenden Dehydratation) delirant wurde. In diesen Phasen verlangte er ausdrücklich nach Flüssigkeit, die ihm auch gereicht wurde. Sobald der Patient wieder orientiert war, zeigte er sich darüber erbost und beklagte, das behandelnde Team habe seinem vorab klar kommunizierten Willen, auf Flüssigkeit und Nahrung verzichten zu wollen, nicht entsprochen.

Trotz medikamentöser und nicht-medikamentöser Massnahmen zur Behandlung und Prophylaxe des Delirs stellten sich immer wieder teilweise sehr ausgeprägte Phasen des deliranten Syndroms ein. Dem Wunsch des Patienten nach Sedation bei erneutem Auftreten des Delirs wurde nach ausführlichen Gesprächen mit dem Patienten (in orientierten Momenten) und Diskussion im multiprofessionellen Team entsprochen. Vereinbart wurde ein niedriges Sedationsniveau, das regelmässig überprüft werden sollte. Zugleich wurden regelmässige Aufwachversuche vereinbart, um den aktuellen Willen des Patienten bzgl. FVNF wiederholt zu überprüfen. Im Verlauf der nächsten Tage reduzierte sich der Allgemeinzustand des Patienten rasch, und er verstarb ruhig und gut symptomkontrolliert.

\section{Ethische Fallbesprechung}

Aufgrund der Komplexität des Falles und der existentiellen Konsequenzen wurde noch während des stationären Aufenthalts des Patienten eine ethische Fallbesprechung mit einem externen Experten geplant. Diese fand in offener, aufmerksamer und kollegialer Atmosphäre statt.

Viele Beteiligte waren der Ansicht, dass der anfängliche Entscheid zur Aufnahme des Patienten zu hinterfragen sei. Der explizite und konsequent verfolgte Wille des Patienten zum FVNF sei möglicherweise zu wenig ernst genommen worden. Der Patient hätte aus Sicht dieser Teammitglieder daher nicht ins Palliativzentrum, sondern direkt in eine Pflegeinstitution überwiesen werden sollen, wo sein Sterben im Rahmen der allgemeinen Palliative Care gut hätte begleitet werden können. Zur möglicherweise notwendig werdenden Symptomkontrolle wäre der Beizug der spezialisierten Palliative Care (mobiles Palliative-Care-Team) durch die Pflegeeinrichtung denkbar gewesen.

Gleichzeitig bestand jedoch bei allen Beteiligten Einigkeit, dass ein "Abschieben" des Patienten aus dem Palliativzentrum nach begonnener stationärer Betreuung aufgrund der speziellen Lebensgeschichte des Patienten menschlich kaum zumutbar gewesen wäre.

Ausserdem wurde hinterfragt, ob der Patient ausreichend über mögliche Komplikationen eines FVNF (starkes Durstgefühl, Delir etc.) informiert war. Einige Beteiligte vertraten die Meinung, dass der alleinige Verzicht auf Nahrung vermutlich einen zwar längeren, aber besser prognostizierbaren und kontrollierbaren Verlauf ergeben hätte (sogenannter FVN [1]).

Da das behandelnde Palliativzentrum bei der Indikationsstellung für die Sedierung vergleichsweise zurückhaltend ist, wurde die Sedation des Patienten besonders intensiv und differenziert diskutiert. Unstrittig ist, dass eine Sedation zur Unterdrückung von Hungerund Durstgefühlen nicht statthaft gewesen wäre [2].

\section{Unstrittig ist, dass eine Sedation}

zur Unterdrückung von Hunger- und Durstgefühlen nicht statthaft gewesen wäre.

Einig war man sich im Team auch bzgl. der Flüssigkeitsgabe im Delir: Die in der Vernehmlassung befindlichen medizinethischen Richtlinien der SAMW «Umgang mit Sterben und Tod» halten in diesem Zusammenhang fest: «Essen und Trinken darf nicht vorenthalten werden, wenn die Patientin danach verlangt. Dies gilt auch, wenn die Patientin in ihrer Patientenverfügung das Gegenteil festgehalten hat" [2]. Auch ein deliranter Patient kann (wie auch ein Säugling) darüber urteilen, 
ob er Durst verspürt, und diesen Durst kommunizieren. Diesem Wunsch nach Flüssigkeit muss seitens der Pflege entsprochen werden.

Als besonders belastend empfanden einige Mitglieder des Teams das Gefühl, den Suizid des Patienten "aktiv» unterstützt zu haben. Das Behandlungsteam befand sich in einer dilemmatischen Situation, in der es zwischen dem Selbstbestimmungsrecht des Patienten und dem medizinisch-pflegerischen Auftrag des Lebensschutzes abwägen musste. Diesbezüglich sind im Zentrum neue, multiprofessionelle Richtlinien für den Umgang mit zentralen ethischen Fragen in Arbeit.

\section{Allgemeine medizinethische Reflexion zum FVNF}

Der FVNF wurde in den vergangenen Jahren in diversen wissenschaftlichen und allgemeinen Publikationen beschrieben. Bisweilen ist er als eine zu priorisierende Alternative zum assistierten Suizid dargestellt [3]. Im Zusammenhang solcher Äusserungen wird oft von "Sterbefasten" gesprochen. Dieser Begriff verleiht dem FVNF eine quasi-religiöse Aura (Fasten wird in vielen Religionen als Askeseleistung verstanden). Befürworterinnen des FVNF argumentieren, der FVNF ermögliche im Unterschied zum assistierten Suizid einen natürlichen Sterbeprozess und könne vom Patien-

\section{Als besonders belastend empfanden einige Mitglieder des Teams das Gefühl, den Suizid des Patienten «aktiv» unterstützt zu haben.}

ten ohne Beihilfe von aussen durchgeführt werden [4]. Es ist unseres Erachtens kaum gerechtfertigt, einen Sterbeprozess, der durch den bewussten Verzicht auf Flüssigkeit und Nahrung herbeigeführt wurde, als «natürlich» zu bezeichnen. Nahrungs- und Flüssigkeitsaufnahme gehören mithin zu den "natürlichsten» Lebensvollzügen. Ohnehin ist Natürlichkeit ein aus ethischer Perspektive sehr problematischer Begriff (vgl. naturalistischer Fehlschluss).

Der hier beschriebene Fall zeigt unter anderem, dass im Rahmen des FVNF unter Umständen substantielle
Unterstützung von aussen notwendig sein kann. Involvierte Fachpersonen fragten, in welchem Mass sie zum Suizid des Patienten beigetragen hätten.

Es ist klar, dass Massnahmen zur Symptomkontrolle beim FVNF nicht als aktive Sterbehilfe betrachtet wer-

\section{Der FVNF muss daher als Form der Selbsttötung} verstanden werden.

den können, da der Tod als kausale Folge des Verzichts auf Nahrungs- und Flüssigkeitsaufnahme eintritt. Es kann in diesem Zusammenhang aber auch nicht von der Beendigung einer lebensunterstützenden Massnahme gesprochen werden, wie bei der passiven Sterbehilfe. Der FVNF muss daher als Form der Selbsttötung verstanden werden. Die Massnahmen zur Symptomkontrolle sind in diesem Zusammenhang nicht als Beihilfe zum Suizid zu bezeichnen.

Dennoch kann sich, wie der vorliegende Fall zeigt, bei betreuenden Fachpersonen die belastende Frage aufdrängen, ob und inwiefern sie einen Suizid ermöglicht haben.

Fazit: Der FVNF als Form der Selbsttötung soll hier nicht moralisch beurteilt werden. Die angestellten Überlegungen wollen jedoch deutlich machen, dass eine moralische Priorisierung des FVNF gegenüber dem assistierten Suizid ethisch nicht zwingend schlüssig ist. Ferner ist zu beachten, dass ein FVNF mit teilweise gravierenden Belastungen für die Patienten sowie komplexen ethischen, persönlichen und versorgungstechnischen Herausforderungen für die Betreuenden und ihre Institutionen einhergehen kann.

Literatur

1 Fehn S, Fringer A. Notwendigkeit, Sterbefasten differenzierter zu betrachten. SÄZ. 2017;98(36):1161-3.

2 Vgl. die in der Vernehmlassung befindlichen medizinethischen Richtlinien der SAMW «Umgang mit Sterben und Tod», S. 18.

3 Zum Beispiel Hehli S. «Keine Nahrung mehr aufzunehmen, ist ein natürlicher Weg des Sterbens.» https://www.nzz.ch/schweiz/ sterbefasten-ein-ausweg-aus-der-demenz-ld.148715 (Stand: 10.4.2018).

4 Zum Beispiel Klein Remane U. Der freiwillige Verzicht auf Nahrung und Flüssigkeit. Thema im Fokus. 2016;128:27. 